\title{
WSI-Mindestlohnbericht 2019: Zeit für kräftige Lohnzuwächse und eine europäische Mindestlohnpolitik
}

\author{
Zu Beginn des Jahres 2019 wurden die Mindestlöhne in den meisten Ländern der \\ Europäischen Union (EU) wieder erhöht. Die mittlere Erhöhung gegenüber dem \\ Vorjahr liegt dabei bei 4,8\%. Damit setzt sich der Trend hin zu einer dynamischeren \\ Mindestlohnentwicklung weiter fort. Getragen wird diese Entwicklung von hohen \\ Zuwachsraten in Osteuropa sowie Bestrebungen in Ländern wie Spanien, das \\ Mindestlohnniveau strukturell anzuheben. Hinzu kommt die Debatte zur Rolle \\ von Mindestlöhnen für ein soziales Europa, die im diesjährigen Europawahlkampf \\ zunehmend an Bedeutung gewinnen wird. Schließlich hat die Bundesregierung bereits \\ angekündigt, die Entwicklung einer europäischen Mindestlohnpolitik zu einem \\ Schwerpunkt ihrer EU-Ratspräsidentschaft im zweiten Halbjahr 2020 zu machen.
}

THORSTEN SCHULTEN, MALTE LÜBKER

\section{Einleitung}

Der vorliegende WSI-Mindestlohnbericht gibt einen Überblick über die aktuelle Entwicklung der Mindestlöhne inner- und außerhalb Europas und schreibt damit die seit 2009 laufende jährliche Berichterstattung fort. Diese beruht auf der WSI-Mindestlohndatenbank, die Zeitreihen für inzwischen 37 Länder enthält. ${ }^{1}$ Hierzu gehören alle 22 der 28 EU-Mitgliedsländer, in denen es gesetzliche Mindestlöhne gibt. Hinzu kommen sieben Anrainerstaaten der EU (Albanien, Mazedonien, Moldawien, Russland, Serbien, Türkei und die Ukraine) sowie acht außereuropäische Industrie- und Schwellenländer (Argentinien, Australien, Brasilien, Japan, Kanada, Korea, Neuseeland und die USA). Neben der nominalen Entwicklung werden durchgängig auch die realen, also inflationsbereinigten Trends erfasst. Um die Höhe der länderspezifischen

Die WSI-Mindestlohndatenbank ist online unter www.wsi. de/mindestlohndatenbank abrufbar und enthält neben einer interaktiven Karte umfangreiche Tabellen und Grafiken. Die Datenbank ist in deutscher und englischer Sprache verfügbar. Soweit im Folgenden einzelne Daten nicht gesondert ausgewiesen werden, sind sie dieser Datenbank entnommen.
Mindestlöhne vergleichen zu können, werden sowohl die tatsächlichen Wechselkurse verwendet als auch die Kaufkraftstandards und es wird der Abstand der Mindestlöhne zu den Median- und Durchschnittslöhnen des jeweiligen Landes analysiert.

\section{Mindestlöhne in Euro zum 1. Januar 2019}

Im internationalen Vergleich weisen die nationalen Mindestlöhne seit jeher große Niveauunterscheide auf: Innerhalb der Europäischen Union lassen sich dabei im Wesentliche drei Gruppen unterscheiden (Abbildung 1) ${ }^{2}$ : Die erste Gruppe umfasst ein Reihe von westeuropäischen Staaten, in denen - mit Ausnahme von Großbritannien -

2 Nicht berücksichtigt werden in diesem Report diejenigen EU-Staaten, die wie Österreich, Italien und die nordeuropäischen Länder über keinen allgemeinen gesetzlichen Mindestlohn verfügen. In diesen Ländern besteht zumeist eine sehr hohe Tarifbindung, sodass eine weitgehend flächendeckende Mindestlohnsicherung durch Tarifverträge erfolgt, die in der Regel oberhalb der gesetzlichen 
der Mindestlohn (zumeist deutlich) oberhalb von $9 €$ pro Stunde liegt. Den Spitzenplatz nimmt mit 11,97€ das Großherzogtum Luxemburg ein. An zweiter Stelle liegt Frankreich, das seit dem 1. Januar 2019 mit 10,03€ erstmals die Zehn-Euro-Marke überschritten hat. Dahinter kommen die Niederlande $(9,91 €)$, Irland $(9,80 €)$ und Belgien $(9,66 €)$ mit Werten, die sich ebenfalls deutlich auf die Zehn-Euro-Marke zubewegen. Deutschland zeigt mit einem Mindestlohn von 9,19€ bereits einen erheblichen Abstand zu den übrigen westeuropäischen Staaten. Lediglich Großbritannien weist mit $8,85 €$ einen noch niedrigeren Wert auf. Allerdings wird der in Euro ausgedrückte Wert des britischen Mindestlohns stark vom Wechselkurs des britischen Pfundes beeinflusst, das im Zuge des Brexit-Votums vom Juni 2016 gegenüber dem Euro stark abgewertet wurde. Hinzu kommt, dass in Großbritannien bereits für den 1. April 2019 eine weitere kräftige Erhöhung beschlossen wurde, wonach der britische Mindestlohn nach dem hier zugrunde gelegten Wechselkurs des Vorjahres auf $9,28 €$ ansteigt.

Die zweite, mittlere Gruppe umfasst mit Spanien $(5,45 €)$, Slowenien $(5,10 €)$ und Malta $(4,40 €)$ lediglich drei Länder mit einem Mindestlohnniveau zwischen 4 und $8 €$ pro Stunde. Die größte Gruppe besteht aus insgesamt elf EU-Staaten mit Mindestlöhnen zwischen 2 und $4 €$. Zu dieser Gruppe gehören neben Portugal $(3,61 €)$ und Griechenland (3,39€, ab 1.2.2019: 3,76€) ausschließlich osteuropäische Länder, von denen Litauen $(3,39 €)$, Estland $(3,21 €)$, Tschechien $(3,11 €)$ und Polen $(3,05 €)$ mittlerweile die Drei-Euro-Marke überschritten haben. Unterhalb von drei Euro liegen die Mindestlöhne nach wie vor in der Slowakei $(2,99 €)$, Kroatien $(2,92 €)$, Ungarn $(2,69 €)$, Rumänien $(2,68 €)$ und Lettland $(2,54 €)$. Absolutes Schlusslicht ist schließlich Bulgarien, dessen Mindestlohn mit $1,72 €$ sogar noch unterhalb von $2 €$ liegt.

Der bulgarische Mindestlohn ist eher mit den Mindestlöhnen anderer osteuropäischer Anrainerstaaten außerhalb der EU vergleichbar, wie etwa Serbien $(1,77 €)$, Mazedonien $(1,63 €)$ oder Albanien $(1,17 €){ }^{3}$ Etwas höher liegt der Mindestlohn mit 2,30€ in der Türkei. Die niedrigsten Mindestlöhne finden sich hingegen mit jeweils $0,78 €$ in Moldawien und der Ukraine sowie Russland mit o,88€. Im Falle Russlands existieren allerdings neben dem nationalen Mindestlohn zahlreiche regionale und lokale Mindestlöhne, die insbesondere in den großen Städten wie z. B. Moskau $(1,47 €)$ oder St. Petersburg $(1,41 €)$ auf deutlich höherem Niveau liegen.

Auch außerhalb Europas bestehen im Hinblick auf die Höhe des Mindestlohns große Unterschiede. Der mit Abstand höchste Mindestlohn existiert in Australien, wo er mit umgerechnet $11,98 €$ auf einem mit dem höchsten europäischen Mindestlohn in Luxemburg vergleichbaren Niveau liegt. Ein Mindestlohn auf westeuropäischem Niveau findet sich auch in Neuseeland, wo er Anfang 2019 bei $9,67 €$ liegt und zum 1. April 2019 auf umgerechnet $10,37 €$ angehoben wird.

Relativ hoch ist der Mindestlohn mit 8,59€ auch noch in Kanada. Allerdings handelt es sich bei diesem Wert um einen mit der Beschäftigtenanzahl gewichteten Durchschnitt regionaler Mindestlöhne, da Kanada über keinen nationalen Mindestlohn verfügt, sondern verschiedene Mindestlöhne auf der Ebene der Provinzen festgelegt werden. Die regionalen Mindestlöhne schwanken dabei zwischen 7,19€ in der Provinz Nova Scotia und 9,81€ in der Provinz Alberta. Ein ähnliches System mit regionalen Mindestlöhnen, die von den einzelnen Präfekturen bestimmt werden, findet sich auch in Japan. Der gewichtete nationale Durchschnitt liegt hier bei $6,70 €$, wobei die Hauptstadt Tokyo mit 7,55€ den höchsten regionalen Mindestlohn aufweist.

Nach kräftigen Erhöhungen liegt der nationale Mindestlohn in Korea mit 6,43€ jetzt nahe am japanischen Niveau. Beide asiatischen Länder haben mittlerweile die USA überholt, wo der landesweite Mindestlohn von 7,25 US $\$(6,14 €)$ bereits seit 2009 nicht mehr erhöht wurde. Schließlich liegt der Mindestlohn in den beiden lateinamerikanischen Schwellenländern Brasilien und Argentinien bei $1,05 €$ bzw. 1,72€ und damit auf einem Niveau, das mit den osteuropäischen EU-Anrainerstaaten vergleichbar ist.

In den USA verfügen allerdings insgesamt 29 US-Bundesstaaten über eigene regionale Mindestlöhne, die oberhalb des nationalen Mindestlohns liegen. ${ }^{4}$ Der höchste Mindestlohn findet sich derzeit mit 13,25 US\$ $(11,22 €)$ im District of Columbia, gefolgt von den Bundesstaaten Kalifornien, Massachusetts und Washington mit jeweils 12 ,oo us $\$(10,16 €)$, Colorado und New York State mit jeweils 11,10 us $\$(9,40 €)$ sowie Arizona und Maine mit jeweils 11,oo us $\$(9,31 €)$. Hinzu kommt noch eine Vielzahl größerer Städte, die zusätzlich noch eigene lokale Mindestlöhne festgelegt haben. Die höchsten Mindestlöhne
Mindestlöhne in Westeuropa liegt (Schulten et al. 2016). In Österreich haben sich Gewerkschaften und Arbeitgeberverbände auf nationaler Ebene darauf verständigt, dass bis zum Jahr 2020 in allen Tarifverträgen die Lohnuntergrenze bei mindestens $1500 €$ im Monat liegen soll. Bei einer durchschnittlichen tarifvertraglichen Wochenarbeitszeit von 39 Stunden (169 Stunden pro Monat) entspricht dies einem Mindestlohn pro Stunde von 8,90€. Berücksichtigt man darüber hinaus, dass in Österreich 14 Monatsgehälter obligatorisch sind, so liegt ein tarifvertraglicher Mindest- lohn von $1500 €$ pro Monat de facto bei $10,35 €$ pro Stunde.

3 Bei Serbien und Mazedonien handelt es sich um geschätzte Werte, da der Mindestlohn in diesen Ländern nicht als Brutto-, sondern als Nettolohn festgelegt wird.

4 Vgl. hierzu auch die Internetdatenbank des Economic Policy Institute mit alle Daten zu regionalen und lokalen Mindestlöhnen in den USA: https://www.epi.org/minimum-wage-tracker 
finden sich im US-Bundesstaat Washington in den Städten SeaTac mit 16,09 US $\$(13,62 €)$ und Seattle mit 16,00 US $\$$ $(13,55 €)$. Beide Städte waren die ersten, in denen die von Gewerkschaften und anderen sozialen Bewegungen seit Beginn des Jahrzehnts geführte Kampagne „Fight for Fifteen" Erfolg hatte (Rolf 2016) und die mittlerweile sogar über dieses Ziel hinausgegangen sind. Darüber hinaus gibt es eine wachsende Zahl von US-Städten, die mittlerweile einen lokalen Mindestlohn von 15,00 US\$ $(12,70 €)$ eingeführt haben, darunter z. B. New York City und San Francisco.

\section{Mindestlöhne in Kaufkraftstandards zum 1. Januar 2019}

Die Aussagekraft eines internationalen Vergleichs von Mindestlöhnen umgerechnet in Euro ist in mehrfacher Hinsicht eingeschränkt. Zum einen wird der Vergleich bei Staaten außerhalb des Euroraums mitunter stark von Wechselkursschwankungen beeinflusst. ${ }^{5}$ So können Aufoder Abwertungen nationaler Währungen den in Euro ausgedrückten Wert des Mindestlohns künstlich höher oder niedriger erscheinen lassen. In den letzten Jahren wurden vor allem die nationalen Währungen Großbritanniens, Argentiniens, Brasiliens, Russlands und der Türkei relativ stark abgewertet, sodass das Mindestlohnniveau in diesen Ländern ausgedrückt in Euro deutlich unterbewertet ist.

Darüber hinaus wird mit einem in Euro ausgewiesenen Vergleich der Mindestlöhne davon abstrahiert, dass es zwischen den Ländern große Unterschiede bei den Lebenshaltungskosten gibt und damit die Kaufkraft des Mindestlohns stark variieren kann. Um diese Effekte zumindest näherungsweise auszugleichen, werden im Rahmen der WSI-Mindestlohnberichte die nationalen Mindestlöhne immer auch in Kaufkraftstandards (KKS) umgerechnet. Neben Eurostat stehen hierzu die KKS-Umrechnungskurse der Weltbank zur Verfügung. Letztere haben den Vorteil, dass sie für alle betrachteten Länder zur Verfügung stehen. Die folgenden Abschnitte verwenden deshalb durchgängig die KKS-Kurse der Weltbank. ${ }^{6}$

5 In der WSI-Mindestlohndatenbank werden die in nationalen Währungen ausgedrückten Mindestlöhne grundsätzlich zum durchschnittlichen Jahreswechselkurs des Vorjahres (in der aktuellen Version des Jahres 2018) in Euro umgerechnet. Dies hat den Vorteil, dass die Volatilität der Wechselkurse durch einen längeren Referenzzeitraum teilweise ausgeglichen wird. Die in den jährlichen WSI-Mindestlohnberichten ausgewiesenen Mindestlöhne in Euro sind deshalb nicht vergleichbar, da ihnen jeweils unterschiedliche Wechselkurse zugrunde liegen. Für Zeitreihenanalysen

\section{ABBILDUNG 1}

\section{Gesetzliche Mindestlöhne, Stand 1. Januar 2019}

Angaben in Euro pro Stunde

unter $2 € \quad 2$ bis $3,99 € \square 4$ bis $7,99 € \square$ mehr als $8 €$

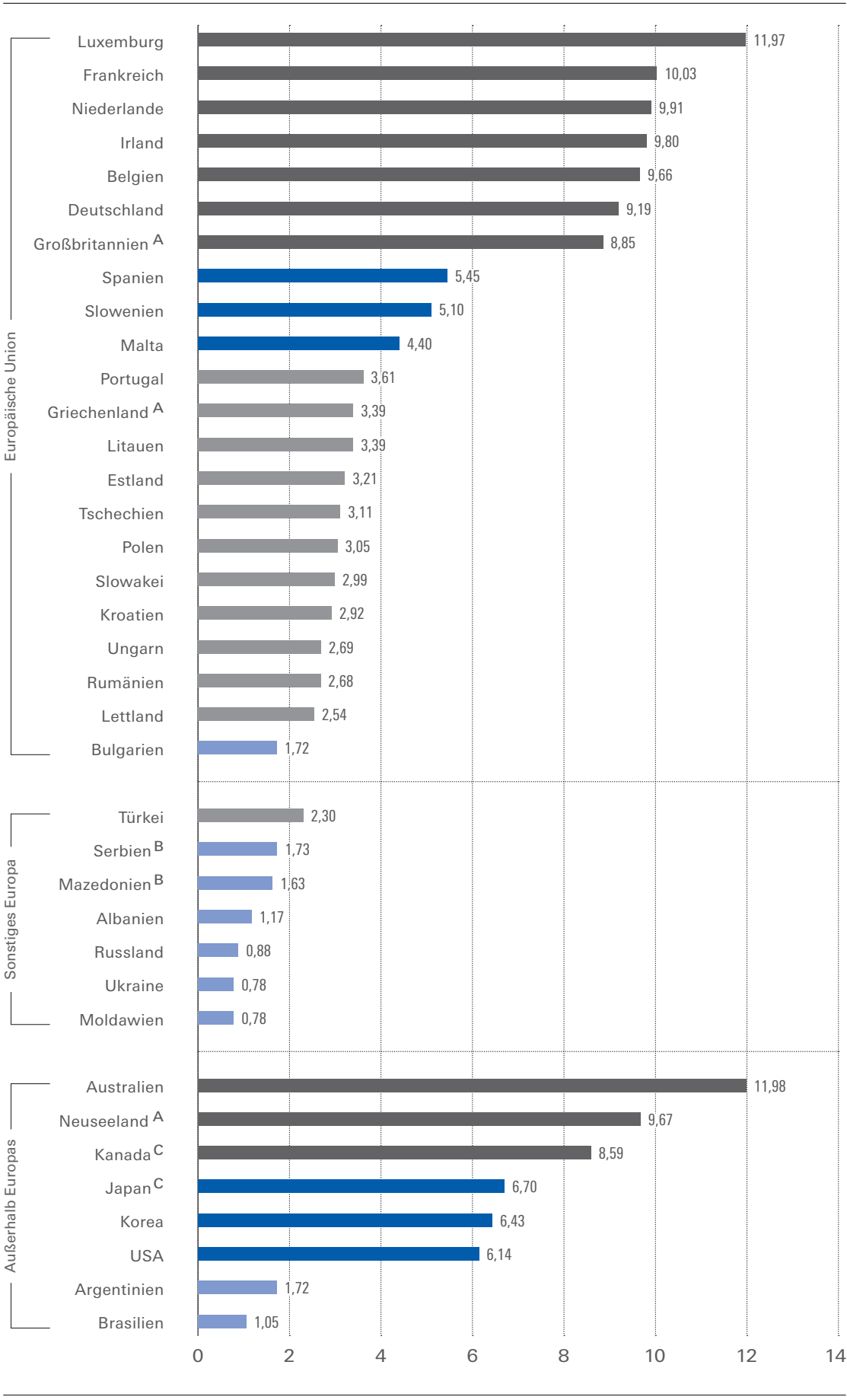

Anmerkungen: Umrechnung in Euro anhand des Durchschnittskurses des Jahres 2018.

A ab 1. 2. 2019: Griechenland 3,76€; ab 1. 4. 2019: Großbritannien 9,28€; Neuseeland: 10,37€

B Geschätzt, da Mindestlohn als Nettolohn festgelegt wird.

C Gewichteter Durchschnitt der regionalen Mindestlöhne.

Quelle: WSI-Mindestlohndatenbank 2019 


\section{ABBILDUNG 2}

Kaufkraft gesetzlicher Mindestlöhne, Stand 1. Januar 2019

Angaben in KKS ${ }^{A}$ auf Euro-Basis, pro Stunde

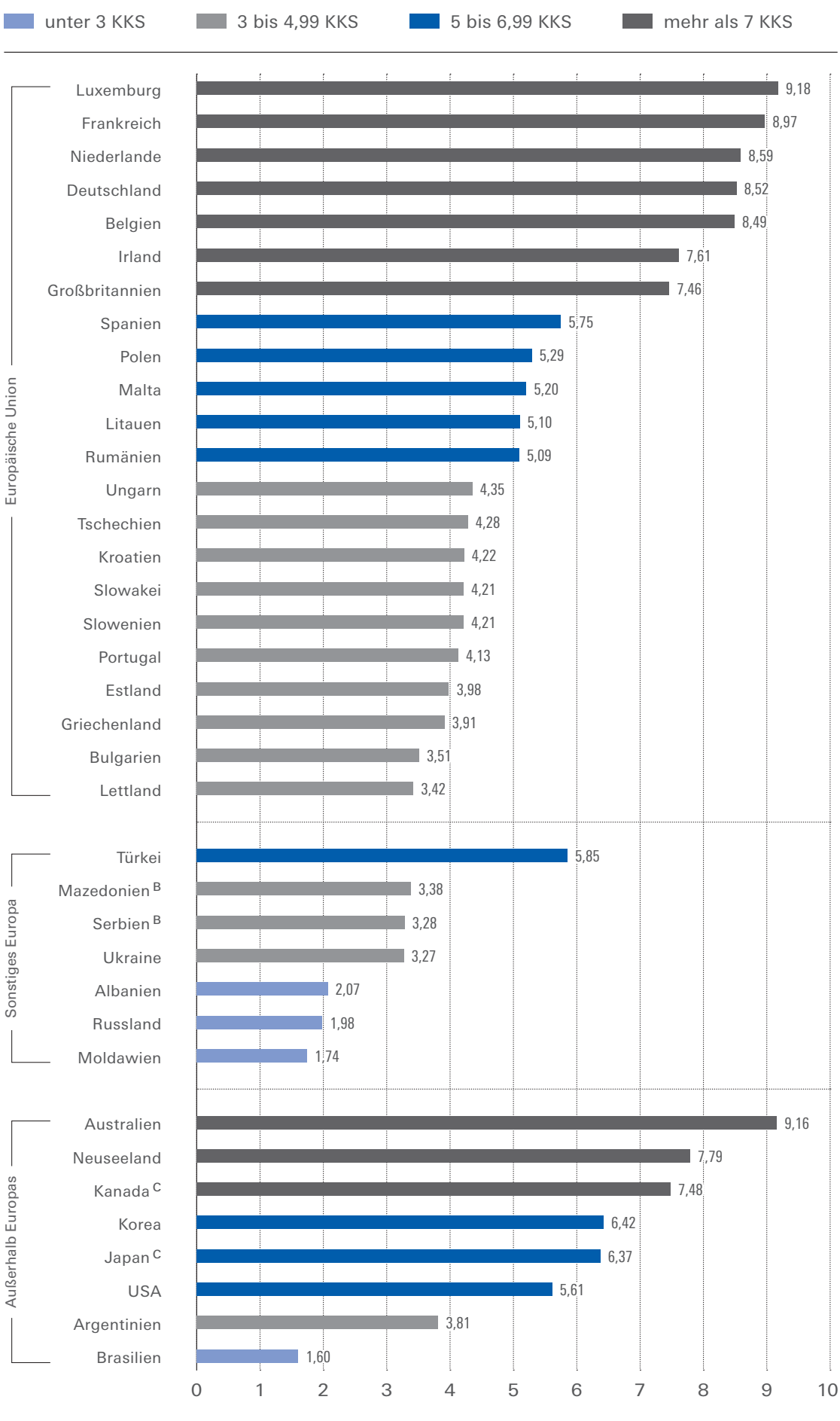

A Umrechnung in KKS auf Euro-Basis aufgrund der von der Weltbank für 2017 ausgewiesenen Kaufkraftparitäten für den privaten Konsum.

B Geschätzt, da Mindestlohn als Nettolohn festgelegt wird.

C Gewichteter Durchschnitt der regionalen Mindestlöhne.

Quelle: WSI-Mindestlohndatenbank 2019
Durch die Berücksichtigung der Kaufkraftunterschiede wird das Mindestlohngefälle innerhalb der Europäischen Union um einiges reduziert, ohne jedoch die substanziellen Niveauunterschiede zwischen den Ländern aufzuheben (Abbildung 2). So ist gemessen in Euro der durchschnittliche Mindestlohn in den EU-Mitgliedstaaten mit den fünf höchsten Mindestlöhnen gut viermal so hoch wie in den Ländern mit den fünf niedrigsten Mindestlöhnen. Auf KKS-Basis reduziert sich der Abstand auf das 2,3-Fache. Demnach reflektiert in der Regel ein niedrigerer Mindestlohn auch niedrigere Lebenshaltungskosten.

Allerdings bleiben auch nach Bereinigung um das nationale Preisniveau erhebliche Unterschiede bestehen und es lassen sich nach wie vor drei Länder-Cluster identifizieren: eine westeuropäische Gruppe mit KKS zwischen $7,46 €$ in Großbritannien und 9,18€ in Luxemburg, eine Gruppe mit süd- und osteuropäischen Ländern mit KKS zwischen 5,09€ in Rumänien und 5,81€ in Slowenien sowie eine ebenfalls aus süd- und osteuropäischen Staaten bestehende Gruppe mit KKS zwischen 3,42€ in Lettland und $4,35 €$ in Ungarn. Während vor zehn Jahren zu Beginn der WSI-Mindestlohnberichterstattung im Jahre 2009 die mittlere Gruppe noch ausschließlich aus südeuropäischen und die untere Gruppe aus osteuropäischen Ländern bestand (Schulten 2009), zeigt die heutige Durchmischung beider Gruppen, dass die Mindestlohnentwicklung in einigen osteuropäischen Länder deutlich aufgeholt hat (z. B. in Polen, Litauen und Rumänien), während sie in einigen südeuropäischen Ländern (insbesondere in Griechenland) eher stagnierte.

Mit der Berechnung in KKS kommt es teilweise auch zu erheblichen Verschiebungen in der Rangfolge der einzelnen Länder. So liegt z. B. der griechische Mindestlohn gemessen in Euro trotz langjähriger Stagnation immer noch auf Platz 12, während er gemessen in KKS auf Platz 20 zurückgefallen ist. Umgekehrt liegt der rumänische Mindestlohn innerhalb der EU gemessen in Euro lediglich auf Platz 20, während er gemessen in KKS - angesichts der relativ niedrigen Lebenshaltungskosten - auf Platz 12 vorrückt. Auch Deutschland verbessert sich in der Rangfolge leicht von Platz 6 (gemessen in Euro) auf Platz 4 (gemessen in KKS). Innerhalb der westeuropäischen Spitzengruppe fallen Irland und Großbritannien aufgrund eines höheren Preiseniveaus bei den in KKS gemessenen Mindestlöhnen bereits deutlich hinter die BENELUX-Länder, Frankreich und Deutschland zurück. Auch außerhalb der EU kommt es durch die Berechnung der Mindestlöhne in KKS zu entsprechenden Bereinigungseffekten.

Nutzern beide Umrechnungen zur Verfügung, die sich im Ergebnis jedoch nur geringfügig unterscheiden (www.wsi. de/mindestlohndatenbank).
6 Hierzu werden die nationalen Mindestlöhne zunächst in KKS auf Dollar-Basis umgerechnet und dann in einem zweiten Schritt in KKS auf Euro-Basis konvertiert. In der frei zugänglichen WSI-Mindestlohndatenbank stehen den 


\section{Der relative Wert des Mindestlohns}

Mit der Berechnung in Kaufkraftstandards wird ein für den internationalen Vergleich nationaler Mindestlöhne realistischeres Maß gewonnen. Mit dieser Methode kann jedoch nichts darüber ausgesagt werden, inwieweit die Mindestlöhne im jeweiligen nationalen Kontext „angemessen“ sind, d.h. ob sie etwa im Sinne der ILO-Konventionen Nr. 131 über die Festlegung von Mindestlöhnen oder der Europäischen Sozialcharta des Europarates geeignet sind, ein bestimmtes Existenzminimum zu gewährleisten (Zimmer 2018). Die Frage der Existenzsicherung von Mindestlöhnen wird derzeit vor allem im Kontext nationaler Living-Wage-Debatten thematisiert. Hierbei gelangen die Protagonisten zumeist zu dem eher kritischen Ergebnis, dass nationale Mindestlöhne oft keine Living Wages sind, sondern auf einem sehr niedrigen Niveau festgelegt wurden, das den Mindestlohnbeziehern ohne weitere staatliche Zuschüsse keine Existenzsicherung ermöglicht. Für den internationalen Vergleich besteht jedoch die Schwierigkeit, dass es keine allgemein akzeptierte Definition von Living Wages gibt, sondern der Begriff im jeweils nationalen Kontext sehr unterschiedlich definiert wird (Hurley et al. 2018; Schulten/Müller 2017).

Um die Angemessenheit von Mindestlöhnen im internationalen Vergleich zu diskutieren, wird deshalb vielfach

\section{ABBILDUNG 3}

\section{Der relative Wert des Mindestlohns (Kaitz-Index) 2017}

Angaben in Prozent

a) Mindestlohn in Prozent des Medianlohns

unter $40 \quad 40$ bis $49,9 \square 0$ bis $59,9 \square$ u. mehr

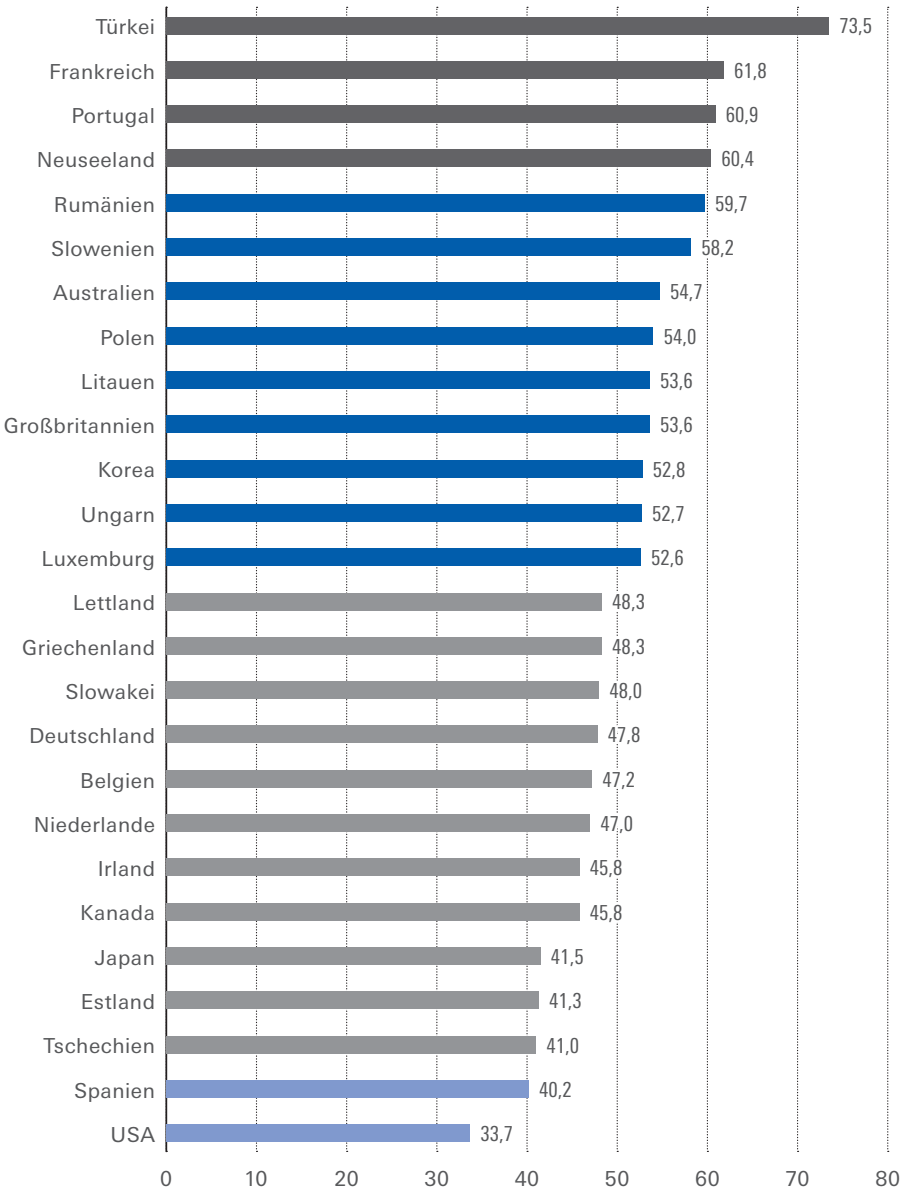

b) Mindestlohn in Prozent des Durchschnittlohns

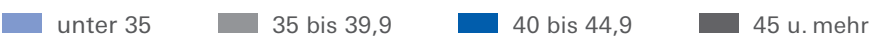

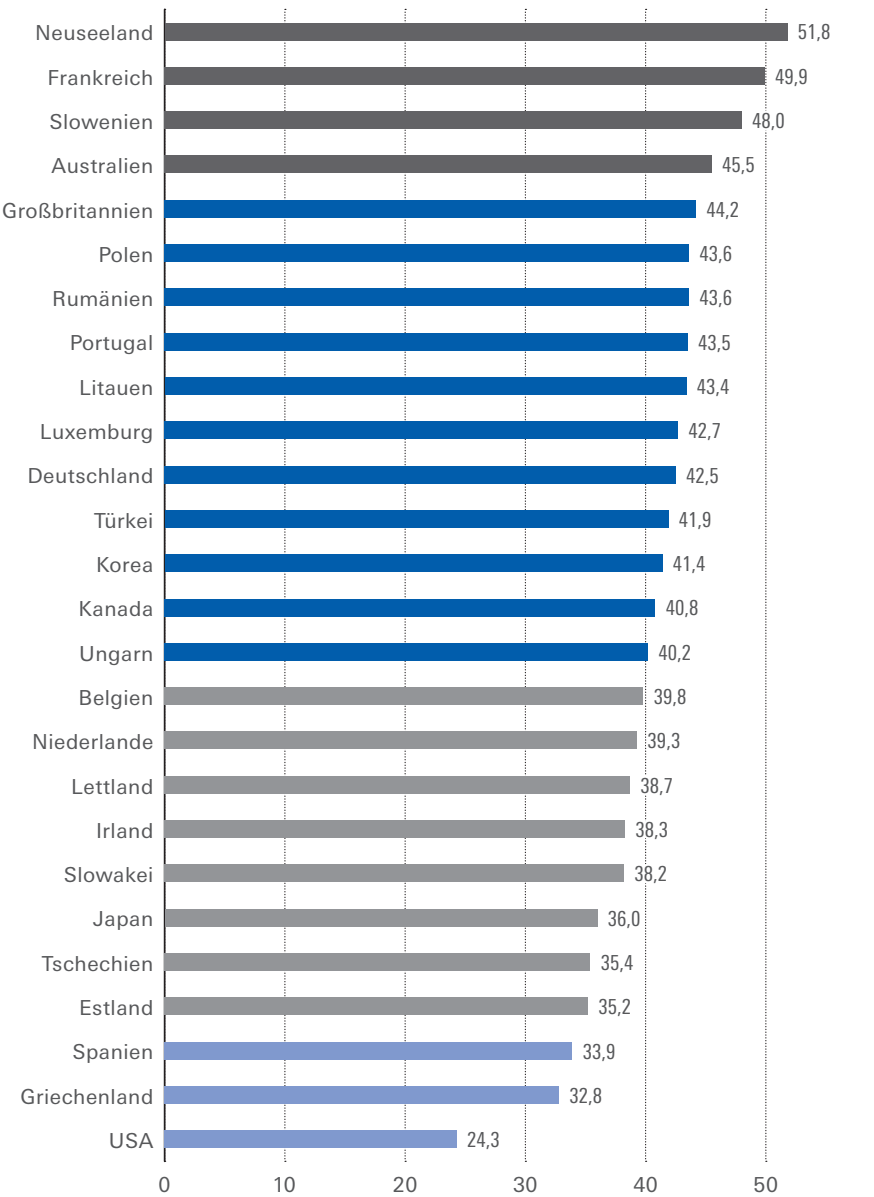

Anmerkung: Daten für folgende Länder sind nicht verfügbar: Albanien, Argentinien, Brasilien, Bulgarien, Kroatien, Malta, Mazedonien, Moldawien, Russland, Serbien und die Ukraine. Alle Werte sind auf eine Nachkommastelle gerundet.

Quelle: OECD Earnings Database 


\section{ABBILDUNG 4}

\section{Entwicklung des relativen Mindestlohnwerts (Kaitz-Index) im EU-Durchschnitt 2000-2017 ${ }^{\mathrm{A}}$}

Mindestlohn in Prozent des Medianlohns

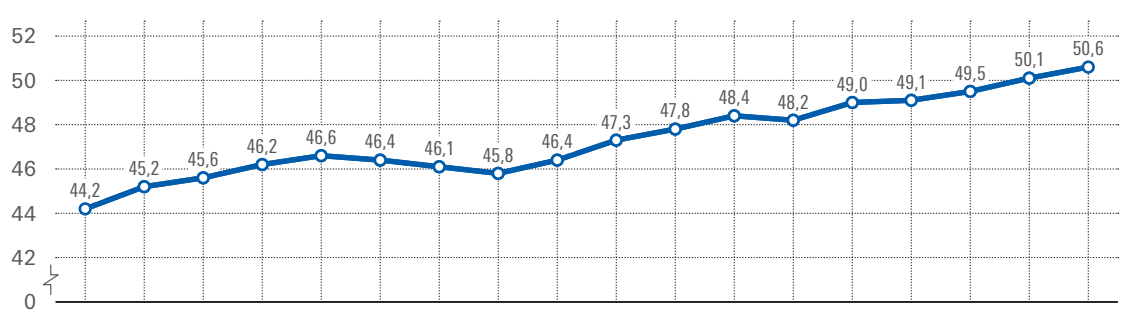

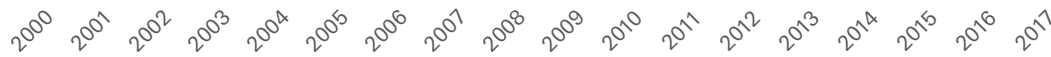

A Ungewichteter Durchschnitt der nationalen Kaitz-Indizes aus 19 EU-Staaten: Belgien, Deutschland, Estland, Frankreich, Griechenland, Großbritannien, Irland, Lettland, Litauen, Luxemburg, Niederlande, Polen, Portugal, Rumänien, Slowakei, Slowenien, Spanien, Tschechien, Ungarn; bis 2004 ohne Deutschland und Slowenien, bis 2014 ohne Deutschland.

Quelle: OECD Earnings Database mutslohn auszugehen ist. Hierzu zählt auch Deutschland, wo der Mindestlohn lediglich bei 47,8\% des Medianlohns liegt. Die mit Abstand niedrigsten Mindestlöhne finden sich neben den USA $(33,7 \%)$ in Tschechien $(41,0 \%)$ und Spanien (40,2\%), wo der Mindestlohn inzwischen aber deutlich angehoben wurde (siehe Abschnitt 5). Im Hinblick auf den Durchschnittslohn variiert der Kaitz-Index zwischen 24,3\% (USA) und 51,8\% (Neuseeland), wobei er sich in der großen Mehrzahl der Länder zwischen 35 und $45 \%$ bewegt.

Das oft nicht-existenzsichernde Niveau der Mindestlöhne hat in zahlreichen Ländern immer wieder politische Auseinandersetzungen hervorgebracht, die zu überdurchschnittlichen Erhöhungen der Mindestlöhne führten. Innerhalb der EU lässt sich demnach seit Beginn der 2oooer Jahre ein Trend hin zu einem höheren Mindestlohnniveau beobachten (Abbildung 4): So stieg der Kaitz-Index im (ungewichteten) EU-Durchschnitt von $44,2 \% \mathrm{im} \mathrm{Jahr}$ 2000 auf 50,6\% im Jahr 2017.

\section{Aktuelle Entwicklung der Mindestlöhne} den relativen Wert des Mindestlohns, indem er dies Relation zu den tatsächlich gezahlten Löhnen und Gehältern eines Landes setzt. In seinem ursprünglichen Aufsatz verwendete Kaitz dazu den Durchschnittslohn (d.h. das arithmetische Mittel), inzwischen hat sich aber weitgehend der Medianlohn als Bezugsgröße durchgesetzt. Letzterer hat den Vorteil, dass er genau die Mitte der Lohnverteilung angibt und deshalb von extrem niedrigen und hohen Werten weitgehend unbeeinflusst ist. In Abbildung 3 wird der Kaitz-Index nach beiden Berechnungsmethoden ausgewiesen, wofür auf die Lohndatenbank der OECD zurückgegriffen wird. ${ }^{7}$

Für die 26 Länder, für die die aktuellsten Daten aus dem Jahr 2017 verfügbar sind, reicht die Spannweite des Kaitz-Indexes von 33,7\% (USA) bis zu 73,5\% (Türkei) des Medianlohns. Relativ hohe Mindestlöhne mit einem Kaitz-Index von über $60 \%$ des Medianlohns finden sich auch in Frankreich (Kaitz-Index: 61,8\%), Portugal (60,9\%) und Neuseeland $(60,4 \%)$. Knapp unterhalb der 6o \%-Marke liegen Rumänien (59,7\%) und Slowenien (58,2\%). Insgesamt bleiben jedoch auch die Länder mit relativ hohen Mindestlohnwerten unter der Niedriglohnschwelle, die nach der OECD-Definition im Allgemeinen bei zwei Dritteln des Medianlohns gesetzt wird.

In 22 von 26 Ländern lagen die Mindestlöhne im Jahr 2017 unterhalb der 60\%-Schwelle, sodass in Analogie zur Armutsforschung von armutsgefährdenden Löhnen gesprochen werden kann. In 16 Ländern wird sogar die Grenze von $50 \%$ des Medianlohns unterschritten, womit in diesen Fällen von einem nicht-existenzsichernden Ar-
In der Mehrzahl der Staaten wird der Mindestlohn jährlich angepasst, und zwar in der Regel zum 1. Januar eines Jahres. Von den 37 in der WSI-Mindestlohndatenbank erfassten Ländern haben 28 ihre Mindestlöhne zuletzt am 1. Januar 2019 erhöht (Tabelle 1). Weitere sechs Staaten haben die Erhöhung im Laufe des Jahres 2018 vorgenommen, während lediglich drei Staaten ihre Mindestlöhne nicht erhöht haben.

Die größte nominale Erhöhung gab es in Litauen mit $38,4 \%$, gefolgt von der Türkei mit $26,0 \%$, Spanien mit 22,3\% sowie Argentinien und Russland mit jeweils 18,9\%. Die Gründe für die außerordentlich großen Mindestlohnerhöhungen waren dabei durchaus verschieden. Während in Argentinien, Russland und der Türkei die hohen $\mathrm{Zu}-$ wachsraten vor allem eine Reaktion auf die hohen Inflationsraten in diesen Ländern darstellten, wurden die kräftigen Erhöhungen in Spanien mit dem Ziel durchgeführt, das Mindestlohnniveau strukturell anzuheben. Eine ähnliche Zielsetzung findet sich auch in Kanada, Korea sowie zahlreichen osteuropäischen Staaten, die ebenfalls alle hohe Steigerungsraten verzeichnen, die sich nomi-

7 Auch wenn die Lohndatenbank der OECD über die Jahre immer wieder erweitert und verbessert wurde, ist die Vergleichbarkeit der Daten aufgrund unterschiedlicher nationaler Quellen und Erfassungsmethoden nicht immer vollständig gegeben (OECD 2012). 
nal zwischen 7 und $12 \%$ und real zwischen 5 und $10 \%$ bewegen.

Eine zweite Ländergruppe, zu der neben Ländern wie Brasilien, Großbritannien, Luxemburg, Australien, Portugal, Japan und Irland auch Deutschland gehört, weisen eher mittlere nominale Mindestlohnzuwächse zwischen 2,5 und $5 \%$ aus, die real zu Lohnerhöhungen zwischen 1 und $2 \%$ führen. Im Fall Deutschlands muss jedoch bedacht werden, dass Deutschland das einzige EU-Land ist, in dem regulär nur eine zweijährige Erhöhung vorgesehen ist. Die jetzige Mindestlohnerhöhung von nominal 4,0\%, real 2,1\% ist also vor dem Hintergrund zu sehen, dass es im Vorjahr 2018 keine Mindestlohnerhöhung gegeben hat.

In einigen wenigen Ländern wie z. B. Belgien, die Niederlande oder Frankreich lag die Erhöhung der Mindestlöhne bei $2 \%$ oder weniger, sodass die Mindestlohnbezieher nach Abzug der Inflationsrate sogar leichte Reallohnverluste hinnehmen mussten. Die drei Länder, die ihre Mindestlöhne überhaupt nicht angehoben haben, sind Lettland, Griechenland und die USA. Griechenland war es seit 2012 von der Troika untersagt worden, seinen Mindestlohn anzuheben. Nach Beendigung des internationalen Kreditprogramms hat die griechische Regierung nun beschlossen, den Mindestlohn zum 1. Februar 2019 um $11 \%$ auf $3,76 € \mathrm{zu}$ erhöhen. Er liegt damit jedoch immer noch deutlich unterhalb des Niveaus des Jahres 2012, als er bereits einen Wert von $4,34 €$ erreicht hatte. ${ }^{8}$

In den USA wiederum scheiterten nunmehr seit gut zehn Jahren alle Initiativen, den nationalen Mindestlohn anzuheben, an den politischen Mehrheiten im Kongress, der diesen Schritt in Form eines Gesetzgebungsverfahrens durchführen muss (siehe dazu Levin-Waldman 2018). Im Januar 2019 haben die Demokraten zum wiederholten Mal eine „Raise the Wage Act“ vorgelegt. Im jüngsten Entwurf ist vorgesehen, den nationalen Mindestlohn schrittweise bis zum Jahr 2024 auf 15,00 US\$ (12,70€) anzuheben. ${ }^{9}$ Unabhängig von der Stagnation auf Bundesebene wurden in mehr als der Hälfte aller US-Bundestaaten regionale Mindestlöhne erhöht, die mittlerweile oft deutlich oberhalb des nationalen Mindestlohns liegen (vgl. Abschnitt 2).

Insgesamt ist innerhalb der EU bereits seit einigen Jahren ein Trend hin zu deutlich höheren Mindestlohnsteigerungen zu beobachten, der sich auch 2019 weiter fortgesetzt hat. Abbildung 5 bildet die Entwicklung in der EU insgesamt $a b$ und verwendet hierfür die mittlere Steigerung der Mindestlöhne, also den gegen extreme Länderwerte unempfindlichen Medianwert. Jeweils die Hälfte der betrachteten Länder weisen also Erhöhungen auf, die über bzw. unter diesem Wert liegen. Die Zahlen zeigen, dass die schon seit 2017 zu beobachtete neue Mindestlohndynamik auch 2019 mit einer nominalen Erhöhung von 4,8 \% weiter anhält. Real bewegt sich die Erhöhung mit 2,7\% ebenfalls auf dem relativ hohen Niveau des Vorjahres. Nach der schwachen Entwicklung der Jahre 2009 bis 2013, in denen die Mindestlohnerhöhungen oft nicht einmal die Preissteigerungen auffangen konnten, steigen die Mindest-
TABELLE 1

Entwicklung gesetzlicher Mindestlöhne, 2019

Veränderung am 1. Januar 2019

gegenüber dem Vorjahreszeitpunkt in Prozent ${ }^{\mathrm{A}}$

\begin{tabular}{|c|c|c|c|}
\hline & Nominal & Real & Zuletzt geändert \\
\hline Litauen & 38,4 & 34,9 & 01.01 .2019 \\
\hline Türkei & 26,0 & 8,3 & 01.01 .2019 \\
\hline Spanien & 22,3 & 20,2 & 01.01 .2019 \\
\hline Argentinien & 18,9 & $-11,4$ & 01.01 .2019 \\
\hline Russland & 18,9 & 15,5 & 01.01 .2019 \\
\hline Kanada & 12,6 & 10,1 & 01.01 .2019 \\
\hline Ukraine & 12,1 & 1,0 & 01.01 .2019 \\
\hline Korea & 10,9 & 8,0 & 01.01 .2019 \\
\hline Bulgarien & 9,8 & 7,0 & 01.01 .2019 \\
\hline Moldawien & 9,7 & 5,9 & 01.05 .2018 \\
\hline Serbien & 9,5 & 7,2 & 01.01 .2019 \\
\hline Rumänien & 9,5 & 5,2 & 01.01 .2019 \\
\hline Kroatien & 9,0 & 7,4 & 01.01 .2019 \\
\hline Tschechien & 9,0 & 6,9 & 01.01 .2019 \\
\hline Albanien & 8,3 & 5,9 & 01.01 .2019 \\
\hline Slowakei & 8,3 & 5,6 & 01.01 .2019 \\
\hline Estland & 8,1 & 4,5 & 01.01 .2019 \\
\hline Ungarn & 7,9 & 4,9 & 01.01 .2019 \\
\hline Polen & 7,1 & 5,9 & 01.01 .2019 \\
\hline Slowenien & 5,2 & 3,2 & 01.01 .2019 \\
\hline Neuseeland & 4,8 & 1,9 & 01.04 .2018 \\
\hline Brasilien & 4,6 & 0,9 & 01.01 .2019 \\
\hline Großbritannien & 4,4 & 1,9 & 01.04 .2018 \\
\hline Deutschland & 4,0 & 2,1 & 01.01 .2019 \\
\hline Luxemburg & 3,6 & 1,6 & 01.01 .2019 \\
\hline Australien & 3,5 & 1,5 & 01.07 .2018 \\
\hline Portugal & 3,4 & 2,2 & 01.01 .2019 \\
\hline Japan & 3,1 & 2,1 & 01.01 .2019 \\
\hline Irland & 2,6 & 1,9 & 01.01 .2019 \\
\hline Belgien & 2,0 & $-0,3$ & 01.09 .2018 \\
\hline Malta & 1,9 & 0,2 & 01.01 .2019 \\
\hline Frankreich & 1,5 & $-0,6$ & 01.01 .2019 \\
\hline Mazedonien & 1,5 & $-0,3$ & 01.07 .2018 \\
\hline Niederlande & 1,4 & $-0,2$ & 01.01 .2019 \\
\hline Lettland & 0,0 & $-2,5$ & 01.01 .2018 \\
\hline Griechenland & 0,0 & $-0,8$ & 01.03 .2012 \\
\hline USA & 0,0 & $-2,4$ & 24.07 .2009 \\
\hline
\end{tabular}

A bereinigt um die Entwicklung der nationalen Verbraucherpreise in 2018 (teilweise vorläufige Werte)

Quelle: WSI-Mindestlohndatenbank 2019

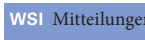

8 Siehe The Guardian vom 28.1. 2019, https://www.thegu ardian.com/world/2019/jan/28/greece-moves-towards-en ding-austerity-with-rise-to-minimum-wage

9 Siehe https://edlabor.house.gov/imo/media/doc/2019-01-16 Raise the Wage Act Section by Section.pdf 
löhne damit insgesamt wieder kräftig. In vielen Ländern wird diese neue Mindestlohndynamik nicht zuletzt von der Tatsache angetrieben, dass ein angemessener existenzsichernder Mindestlohn nach wie vor einer strukturellen Niveauanpassung bedarf.

\section{Ausblick: Auf dem Weg zu einer europäischen Mindestlohnpolitik?}

Anfang 2019 hat Bundesarbeitsminister Hubertus Heil (2019) in einem Interview angekündigt, die Schaffung eines „europäischen Rechtsrahmens für Mindestlöhne und Grundsicherungssysteme“ zu einem Schwerpunkt der deutschen EU-Ratspräsidentschaft im zweiten Halbjahr $2020 \mathrm{zu}$ machen. Eine entsprechende Forderung findet sich bereits im Koalitionsvertrag zwischen CDU/CSU und SPD (CDU et al. 2018, S. 7) und soll nun konkretisiert werden. Bereits im November 2018 hatte sich Bundesfinanzminister Olaf Scholz (2018) in einer Grundsatzrede über Europa ebenfalls für eine europäische Mindestlohninitiative ausgesprochen und dabei betont, dass er „den Vorschlag sehr diskussionswürdig [findet], dass nationale Mindestlöhne etabliert werden, die mindestens 6o Prozent des nationalen Medianlohns betragen“. Schließlich hat die Bundesjustizministerin und SPD-Spitzenkandidatin für die Europawahl, Katarina Barley, angekündigt, den „europäischen Mindestlohn zu einer zentralen Forderung im Wahlkampf zu machen“, da ,jeder Mensch [...] von der eigenen Arbeit leben können [muss], und zwar überall in der EU“ (zit. nach Handelsblatt vom 12. 01. 2019).

Auch andere Parteien greifen das Thema im Europawahlkampf auf. So setzen sich z. B. Bündnis 9o/Die Grünen (2019, S. 75) für „eine Mindestlohnrichtlinie ein, die allen Arbeitnehmer*innen in der EU, entsprechend den Lebenshaltungskosten des jeweiligen Landes, ein auskömmliches Einkommen garantiert". Und die Partei Die Linke tritt für „eine verbindliche europäische Mindestlohnregelung ein“, bei der „alle EU-Staaten [...] dazu verpflichtet [werden], existenzsichernde Mindestlöhne einzuführen, [die] oberhalb von 6o Prozent der mittleren Verdienste in den jeweiligen Ländern liegen" (Die Linke 2018). Schließlich hat auch der Deutsche Gewerkschaftsbund die Forderung nach einem „System europäischer Mindestlöhne und sozialer Grundsicherung “ begrüßt (DGB 2018a). Zuvor hatte der DGB-Bundeskongress den Beschluss gefasst, „den Mindestlohn so weiterzuentwickeln, dass er existenzsichernd“ sei, d.h. „dass jeder alleinstehende Vollzeitbeschäftigte unabhängig werden soll von sozialen Transferleistungen, kein Altersarmutsrisiko mehr hat und von seinem Lohn leben kann" (DGB 2018b, S. 85). Damit liegt der DGB auf der Linie des Europäischen Gewerkschaftsbundes (EGB), der dafür eintritt, dass Min-

\section{ABBILDUNG 5}

\section{Entwicklung der gesetzlichen Mindestlöhne in der EU, 2000-2019}

Angaben in Prozent, mittlere Veränderung zum Vorjahr

— Nominale Entwicklung (Median für die EU)

— Reale Entwicklung (Median für die EU)

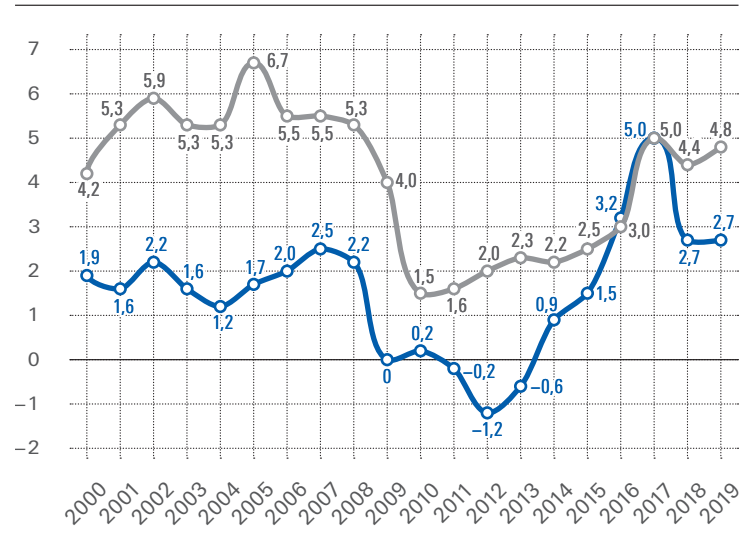

Anmerkungen: Angegeben ist der Medianwert der nationalen Veränderungsraten, jeweils Stand am 1. Januar im Vergleich zum Vorjahreswert. Reale Werte sind preisbereinigt um die Veränderung der nationalen Verbraucherpreise im Vorjahr. Datengrundlage sind jeweils alle EU-Staaten mit einem gesetzlichen Mindestlohn (derzeit 22 Länder).

Quelle: WSI-Mindestlohndatenbank 2019

destlöhne überall in Europa prinzipiell existenzsichernde Living Wages sein sollen. Nach den Vorstellungen des EGB sollen hierfür die Mindestlöhne in ganz Europa auf mindestens $60 \%$ des jeweiligen nationalen Medianlohns angehoben werden (EGB 2017).

Die Debatte über eine europaweit koordinierte Mindestlohnpolitik hat mittlerweile eine relativ lange $\mathrm{Ge}$ schichte, die bis in die frühen 199oer Jahre zurückreicht (Schulten 2008). Sie findet ihre normativen Grundlagen in zahlreichen internationalen und europäischen Vereinbarungen und Deklarationen, in denen das Recht auf eine faire, existenzsichernde Entlohnung als soziales Grundrecht postuliert wird (Zimmer 2018). Zuletzt wurde der Gedanke der Living Wages im Jahr 2017 als einer der 20 Grundsätze in die „Europäische Säule sozialer Rechte“ aufgenommen. Demnach haben alle Arbeitnehmerinnen und Arbeitnehmer das „Recht auf eine gerechte Entlohnung, die ihnen einen angemessenen Lebensstandard ermöglicht" (Europäisches Parlament et al. 2017, Artikel 6).

Im Kern geht es bei der Idee einer europäischen Mindestlohnpolitik darum, überall in Europa das soziale Grundrecht auf eine existenzsichernde Entlohnung in die Praxis umzusetzen. Hierzu sollen auf europäischer Ebene gemeinsame Normen und Ziele für ein angemessenes Mindestlohnniveau formuliert werden, die über die europäischen Koordinierungs- und Governancemechanismen in die nationale Mindestlohnpolitik einfließen. Zur 
Umsetzung einer europäischen Mindestlohnpolitik bietet sich als erster Schritt das Europäische Semester an, wie dies z.B. vom französischen Parlament vorgeschlagen wurde (Cordery 2016). Damit können die juristischen Vorbehalte, die sich aus der Regelungssperre gegenüber Entgeltfragen im EU-Vertrag (Artikel 153, Abs.5) ergeben, zunächst umgangen werden.

Als inhaltliche Norm für eine europäische Mindestlohnpolitik setzt sich in den Debatten auf europäischer Ebene immer mehr die Orientierung des Mindestlohns an einem bestimmten Prozentsatz des nationalen Medianlohns durch, wobei zumeist die 60\%-Marke als Zielgröße definiert wird (Schulten 2015; Schulten et al. 2016). Damit knüpft die europäische Debatte auch direkt an nationale Auseinandersetzungen an, die in vielen Ländern um eine strukturelle Anpassung des Mindestlohns geführt werden. So hat z. B. die britische Regierung im Jahr 2016 die Einführung eines neuen National Living Wage mit der Zielstellung verbunden, diesen bis zum Jahr 2020 auf 60 \% des Medianlohns anzuheben (Department for Business 2015). In Spanien wurde die strukturelle Mindestlohnerhöhung von mehr als $22 \%$ zum Januar 2019 explizit damit begründet, einen wichtigen Schritt auf das 60\%-Ziel zuzugehen (Ministerio de Trabajo, Migraciones y Seguridad Social 2018). Auch in vielen osteuropäischen Ländern wird ein entsprechender Kaitz-Index als Zielmarke für eine strukturelle Mindestlohnerhöhung diskutiert. So hat z.B. der slowakische Ministerpräsident Peter Pellegrini bei der jüngsten Mindestlohnerhöhung angekündigt, „das dynamische Wachstum des Mindestlohns mit dem Ziel fortzusetzen, diesen so anzuheben, dass er mindestens $60 \%$ des nationalen Durchschnittslohns entspricht" (zit. n. The Slovak Spectator vom 6.11. 2018; eigene Übersetzung). Schließlich kann auch die in Deutschland geführte Auseinandersetzung um einen existenzsichernden Mindestlohn gut an die europäische Debatte anknüpfen, zumal die mittlerweile von zahlreichen Protagonisten formulierte Zielgröße von $12 €$ sich nahe an der $60 \%$-Marke des deutschen Medianlohns bewegt.

Die in jüngster Zeit zu beobachtende dynamische Entwicklung der Mindestlöhne macht insgesamt deutlich, dass in vielen europäischen Ländern eine strukturelle Lohnerhöhung angestrebt wird, die den Mindestlohn auf ein angemessenes, existenzsicherndes Niveau anhebt. Ein solcher Ansatz könnte durch eine europäische Mindestlohnpolitik zusätzlich unterstützt und befördert werden und zugleich der Idee eines sozialeren Europas praktische Gestalt verleihen.

\section{LITERATUR}

Bündnis 9o/Die Grünen (2018): Europas Versprechen erneuern, Europawahlprogramm der Partei Bündnis 90/Die Grünen, Berlin, https://www.gruene.de/ fileadmin/user_upload/Dokumente/BgoGRUENE_Europawahlprogramm_2019.pdf CDU, CSU und SPD (2018): Ein neuer Aufbruch für Europa. Eine neue Dynamik für Deutschland. Ein neuer Zusammenhalt für unser Land. Koalitionsvertrag zwischen CDU, CSU und SPD, Berlin, 7. Februar 2018, https://www.bundesregie rung.de/breg-de/themen/koalitionsvertrag-zwischen-cdu-csu-und-spd-1959o6 Cordery, P. (2016): Le salaire minimum au sein de I'Union européenne, Assemblée Nationale Rapport d'Information No. 3856, 21. Juni 2016, http://www. assemblee-nationale.fr/14/pdf/europe/rap-info/i3856.pdf

Department for Business, Economy and Skills (2015): Low pay commission remit 2016, London

DGB (Deutscher Gewerkschaftsbund) (2018a): DGB fordert System europäischer Mindestlöhne und sozialer Grundsicherung, Pressemitteilung vom 28. 11. 2018, https://www.dgb.de/presse/++co++fogf172a-f319-11e8-902b52540088cada

DGB (2018b): Beschlüsse des DGB-Bundeskongresses, Berlin, 13.-17. Mai 2018 Berlin, https://www.dgb.de/uber-uns/dgb-heute/bundeskongress/21-ordent- licher-bundeskongress/dgb-obk-beschluesse-21-parlament-der-arbeit-dgb-bundeskongress-2018

Die Linke (2018): Für ein solidarisches Europa der Millionen, gegen eine Europäische Union der Millionäre, Entwurf des Europawahlprogrammes der Partei Die Linke, Berlin, https://www.die-linke.de/fileadmin/download/parteivor stand/2018-2020/beschluesse/185_Europawahlprogramm_-_Auszug_aus_An tragsheft_I.pdf

EGB (Europäischer Gewerkschaftsbund) (2017): Für eine gemeinsame Strategie zu Niedrig- und Mindestlöhnen, Entschließung der EGB Exekutive vom 15./16. März 2017

Europäisches Parlament/Europäischer Rat/Europäische Kommission (2017): Europäische Säule sozialer Rechte, beschlossen auf dem EU-Sozialgipfel in Göteborg am 17. November 2017, https://ec.europa.eu/commission/sites/beta-po litical/files/social-summit-european-pillar-social-rights-booklet_de.pdf

Heil, H. (2019): Heil will EU-Mindestlohn, Interview mit dem Bundesarbeits- und Sozialminister, in: Neue Osnabrücker Zeitung vom 12. Januar 2019

Hurley, J./ Vacas-Soriano, C. / Muraille, M. / Lantto, E. (2018): Concept and practice of a living wage, Eurofound Research Report, Luxembourg Kaitz, H. (1970): Experience of the past: The national minimum, in: Youth Unemployment and Minimum Wages, Bulletin 1657, US Department of Labor, S. 30-54 Levin-Waldman, O. M. (2018): Restoring the middle class through wage policy. Arguments for a minimum wage, Basingstoke

Ministerio de Trabajo, Migraciones y Seguridad Social (2018): Real Decreto 1462/2018, de 21 de diciembre, por el que se fija el salario mínimo interprofesional para 2019, in: Boletín Oficial del Estado Nr. 312, 27. Dezember, https://www. boe.es/boe/dias/2018/12/27/pdfs/BOE-A-2018-17773.pdf

OECD (Organisation for Economic Co-operation and Development) (2012): Quality review of the OECD database on household incomes and poverty and the OECD earnings database, Part II, Paris

Rolf, D. (2016): The Fight for Fifteen: The right wage for a working America, New York

Scholz, O. (2018): Ein starkes, ein souveränes, ein gerechtes Europa liegt in unserem ureigenen Interesse, Rede an der Humboldt-Universität zu Berlin, 28. November 2018, https://www.bundesfinanzministerium.de/Content/DE/ Re den/2018/2018-11-28-Europarede-HU-Berlin.html

Schulten, T. (2008): Towards a European minimum wage policy? Fair wages and social Europe, in: European Journal of Industrial Relations 14 (4), S. 421-439 Schulten, T. (2009): WSI-Mindestlohnbericht 2009, in: WSI-Mitteilungen 62 (3), S.150-157, https://www.boeckler.de/wsimit_2012_02_schulten.pdf

Schulten, T. (2015): Konturen einer europäischen Mindestlohnpolitik, in: Alemann, U. von / Heidbreder, E. G. / Hummel, H. / Dreyer, D. / Gödde, A. (Hrsg.): Ein soziales Europa ist möglich: Grundlagen und Handlungsoptionen, Wiesbaden, S. $159-182$

Schulten, T./ Müller, T. (2017): Living Wages - normative und ökonomische Gründe für einen angemessenen Mindestlohn, in: WSI-Mitteilungen 70 (7), S. 507-514, https://www.boeckler.de/wsi-mitteilungen_111132_111141.htm Schulten, T. / Müller, T. / Eldring, L. (2016): Für eine europäische Mindestlohnpolitik, in: Müller, T./ Van Gyes, G./ Schulten, T. (Hrsg.): Lohnpolitik unter europäischer Economic Governance, Hamburg, S. 246-274

Zimmer, R. (2018): Das Recht auf ein existenzsicherndes Einkommen als Bestandteil des Unionsrechts?, in: Zeitschrift für europäisches Sozial- und Arbeitsrecht (ZESAR) 17 (4), S. 150-156

\section{AUTOREN}

THORSTEN SCHULTEN, Prof. Dr., ist Wissenschaftler im Wirtschafts- und Sozialwissenschaftlichen Institut (WSI) der Hans-Böckler-Stiftung. Arbeitsschwerpunkte: WSI-Tarifarchiv, Arbeits- und Tarifpolitik in Europa.

thorsten-schulten@boeckler.de

MALTE LÜBKER, Dr., ist Wissenschaftler im Wirtschafts- und Sozialwissen schaftlichen Institut (WSI) der Hans-Böckler-Stiftung. Arbeitsschwerpunkte: Löhne, personale und funktionale Einkommensverteilung und Umverteilung durch den Wohlfahrtsstaat.

malte-luebker@boeckler.de 